\title{
Nanometer Scale Time of Flight Back Scattering Spectrometry in the Helium Ion Microscope
}

\author{
Nico Klingner ${ }^{1}$, Gregor Hlawacek ${ }^{1}$, Rene Heller ${ }^{1}$, Johannes von Borany ${ }^{1}$ and Stefan Facsko ${ }^{1}$ \\ 1. Institute for Ion Beam Physics and Materials Research, Helmholtz Zentrum Dresden Rossendorf, \\ Dresden, Germany
}

Helium Ion Microscopy (HIM) [1] is well known for its high resolution imaging and nano fabrication capabilities. However, in terms of analytic capabilities it lags behind comparable techniques such scanning electron microscopy (SEM) or transmission electron microscopy (TEM). Although several primary and secondary particles are available to date none of them has been exploited in practical way to obtain analytic information. Although electrons are used for imaging they are of limited use due to matrix effects which complicate the elemental analysis. The existing models are based on empirical data and can deliver useful results only for a selected number of materials [2]. Photons have been exploited in the past to obtain information on the sample composition but turned out to be of limited practical use due to the high sensitivity for damage induced by the ion beam [3]. In the past backscattered ions have been used to obtain materials contrast in a qualitative way. Recently, the successful use of sputtered particles for analytic purposes has been demonstrated by adding a sophisticated secondary ion mass spectrometer to the HIM [4].

Here, we present the first successful attempt to use $t$ ime of flight ion backscattering spectrometry (TOFBS) for materials characterization in a HIM [5]. The start signal for the TOF measurements is created by chopping the primary beam of the ion microscope using the built — in blanker and a custom made electronics that allows pulse lengths of $10 \mathrm{~ns}$ to $250 \mathrm{~ns}$. The stop signal is given by the arrival of the backscattered particles at a micro channel plate. The setup has the advantage of providing a high lateral resolution, a good energy resolution and at the same time is minimal invasive to the microscope and therefore not deteriorating the high resolution capabilities of the device when the BS setup is not in use.

TOF-BS spectra of $\mathrm{HfO}_{2}$ on $\mathrm{Si}$ are presented in fig. 1 . The time resolution is limited by the physical length of the microscope blanker to approximately $17 \mathrm{~ns}$ or $5.4 \%$. This value can be decreased to $2.7 \%$ by using a longer flight path. Thanks to a home built scan system to control the beam TOF-BS data can be recorded also in imaging mode. This allows an efficient post acquisition analysis by applying energy filters to extract the elemental distribution. An example is presented in fig. 2. The corresponding bulk BS spectra are color coded to the extraction areas in the secondary electron (left in fig. 2) and total BS image (right in fig. 2). A lateral resolution of $54 \mathrm{~nm}$ has been determined. Although this is not comparable to the native resolution of the tool, the value is close to the physical limit and can be overcome by using correlative approaches in connection with the high resolution SE data available in the HIM. Modifying the sample holder slightly one can also perform TOF-SIMS. The sputtered particles are accelerated towards the stop detector of the TOF setup by means of a high voltage applied to the sample and a grounded grid. TOF-SIMS spectra obtained from the same sample shown in fig. 2 are presented in fig. 3. The usefulness of the combined TOF-SIMS and TOF-BS setup becomes evident by noting that the TOF-SIMS setup fails to detect the gold due to the low sputter and ionization yield of gold. This inherent weakness of SIMS is overcome in this combined setup. 


\section{References:}

[1] Hlawacek, G., et al, Journal of Vacuum Science \& Technology B: Microelectronics and Nanometer Structures 32 (2014), p. 020801.

[2] Ramachandra, R., Griffin, B. J. \& Joy, D. C. Ultramicroscopy 109 (2009), p. 748.

[3] Veligura, V., et al, Journal of Luminescence 157 (2015), p. 321.

[4] Wirtz, T., et al, Nanotechnology 26 (2015), p. 434001.

[5] Klingner, N., et al, Ultramicroscopy accepted (2015).

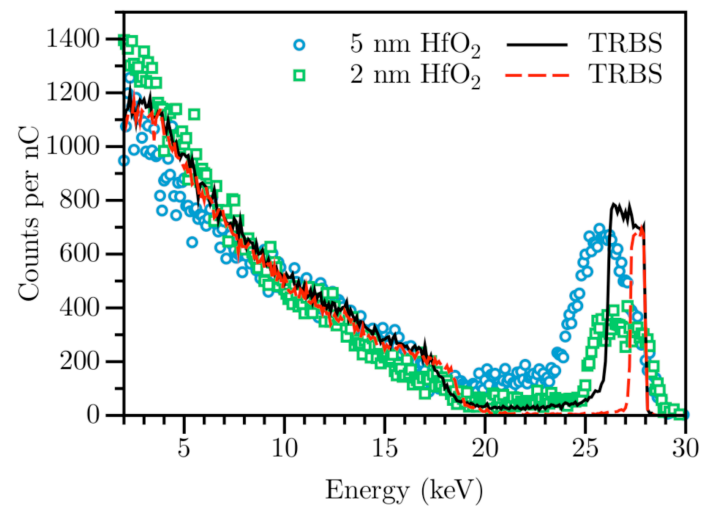

Figure 1: TOF-BS spectra recorded in the HIM. Results for $2 \mathrm{~nm}$ and $5 \mathrm{~nm} \mathrm{HfO}_{2}$ thickness are shown together with TRBS

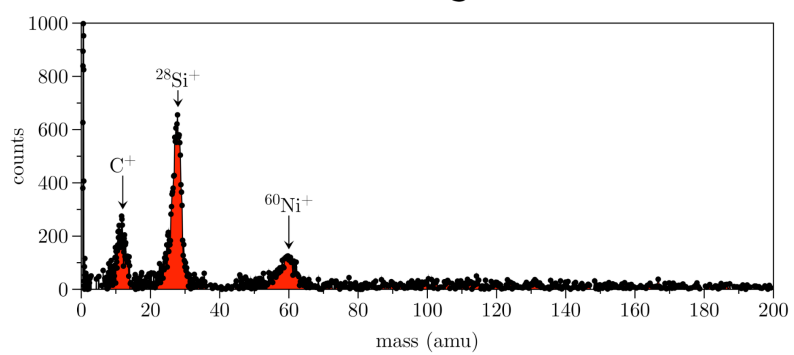

Figure 3: TOF-SIMS spectra obtained showing C, Si and Ni peaks. Au is not present due to low sputter and ionization yield.
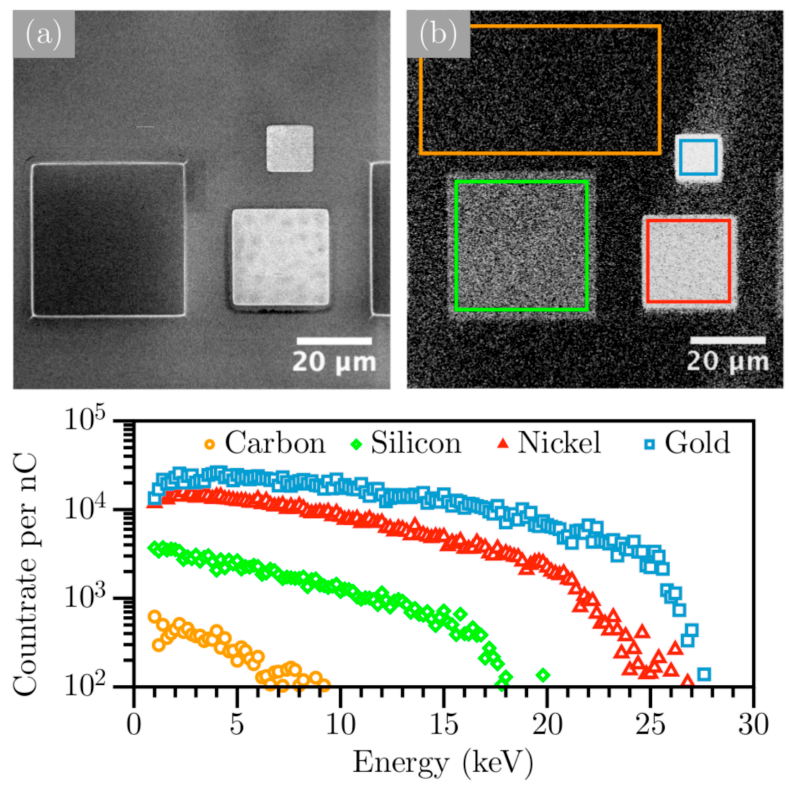

Figure 2: Laterally resolved TOF-BS image. $S E$ mode and TOF BS ion image are presented. Higher gray values in the TOF-BS image corresponds to a shorter TOF. The color coded BS spectra are shown in the bottom row. 